\title{
On the total neighbour sum distinguishing index of graphs with bounded maximum average degree
}

\author{
H. Hocquard ${ }^{\mathrm{a}, 1}$, J. Przybyło ${ }^{\mathrm{b}, 2,3, *}$ \\ ${ }^{a}$ LaBRI (Université de Bordeaux), 351 cours de la Libération, 33405 Talence Cedex, France \\ ${ }^{b}$ AGH University of Science and Technology, al. A. Mickiewicza 30, 30-059 Krakow, Poland
}

\begin{abstract}
A proper total $k$-colouring of a graph $G=(V, E)$ is an assignment $c: V \cup E \rightarrow\{1,2, \ldots, k\}$ of colours to the edges and the vertices of $G$ such that no two adjacent edges or vertices and no edge and its end-vertices are associated with the same colour. A total neighbour sum distinguishing $k$-colouring, or tnsd $k$-colouring for short, is a proper total $k$-colouring such that $\sum_{e \ni u} c(e)+c(u) \neq \sum_{e \ni v} c(e)+c(v)$ for every edge $u v$ of $G$. We denote by $\chi_{\Sigma}^{\prime \prime}(G)$ the total neighbour sum distinguishing index of $G$, which is the least integer $k$ such that a tnsd edge $k$-colouring of $G$ exists. It has been conjectured that $\chi_{\Sigma}^{\prime \prime}(G) \leq \Delta(G)+3$ for every graph $G$. In this paper we confirm this conjecture for any graph $G$ with $\operatorname{mad}(G)<\frac{14}{3}$ and $\Delta(G) \geq 8$.
\end{abstract}

Keywords: Total neighbour sum distinguishing index, maximum average degree, Combinatorial Nullstellensatz, discharging method.

\section{Introduction}

A proper total $k$-colouring of a graph $G=(V, E)$ is an assignment $c$ of colours from the set $\{1,2, \ldots, k\}$ to the edges and the vertices of $G$ such that adjacent edges and vertices are coloured differently and the colour of every edge is distinct from those assigned to its end-vertices. A total neighbour sum distinguishing $k$-colouring of $G$, or tnsd $k$-colouring for short, is its proper total $k$-colouring $c$ such that for every edge $u v \in E$, there is no conflict between $u$ and $v$, i.e., $s(u) \neq s(v)$, where $s(w)$ is the sum of colours taken on the edges incident with $w$ and the colour of the vertex $w$ for $w \in V$. In other words, for every vertex $w \in V$, $s(w)=\sum_{e \in E_{w}} c(e)+c(w)$, where $E_{w}$ is the set of edges incident with $w$ in $G$. We denote by $\chi_{\Sigma}^{\prime \prime}(G)$ the total neighbour sum distinguishing index of $G$, which is the least integer $k$ such that a tnsd $k$-colouring of $G$ exists. The roots of this branch of graph theory date back to the ' 80 s, and the papers [ [6, 7] on degree irregularities in graphs (and multigraphs) and the parameter irregularity strength of a graph. For more details concerning a motivation for investigating integer graph colourings and a few crucial results on the irregularity strength see e.g. [1, 9, 11, 14, 15, 20, 21].

By definition and the requirement of properness of colourings investigated, the total neighbour sum distinguishing index of every graph $G$ is not smaller than $\Delta(G)+1$. The following conjecture was proposed by Pilśniak and Woźniak in [22], where it was also verified for a few classical graph families, including, e.g., complete graphs, bipartite graphs and graphs with maximum degree at most three.

Conjecture $1([\mathbf{2 2}])$. For every graph $G, \chi_{\Sigma}^{\prime \prime}(G) \leq \Delta(G)+3$.

\footnotetext{
${ }^{*}$ Corresponding author

Email addresses: herve.hocquard@labri.fr (H. Hocquard ), jakubprz@agh.edu.pl (J. Przybyło)

${ }^{1}$ Supported by CNRS-PICS Project no. 6367 "GraphPar".

${ }^{2}$ Supported by the National Science Centre, Poland, grant no. 2014/13/B/ST1/01855.

${ }^{3}$ Partly supported by the Polish Ministry of Science and Higher Education.
} 
Note that such postulated upper bound exceeds just by one the bound implied by the famous Total Colouring Conjecture posed by Vizing [26] in 1968 and independently by Behzad [3] in 1965, and concerning proper total colourings (without our additional requirement on sum distinction between adjacent vertices). The both conjectures seem to be very challenging and are in general widely open. The best general result concerning the latter one [18] confirms however the Total Colouring Conjecture up to a (large) additive constant.

The best general upper bound concerning Conjecture 1 implies that $\chi_{\Sigma}^{\prime \prime}(G) \leq(1+o(1)) \Delta$ for every graph $G$ with maximum degree $\Delta$, see [19] and [23]. See also [16, 17, 22, 24] for partial results on this conjecture. In particular Ding et al. first confirmed Conjecture 1 for planar graphs with sufficiently large maximum degree:

Theorem 2 ([16] $)$. Any planar graph $G$ with $\Delta(G) \geq 13$ satisfies $\chi_{\Sigma}^{\prime \prime}(G) \leq \Delta(G)+3$.

This was then improved by Yang et al. in the following form.

Theorem 3 ([27]). Any planar graph $G$ satisfies $\chi_{\Sigma}^{\prime \prime}(G) \leq \max \{\Delta(G)+2,13\}$.

Let $\operatorname{mad}(G)=\max \left\{\frac{2|E(H)|}{|V(H)|}, H \subseteq G\right\}$ be the maximum average degree of a graph $G$, where $V(H)$ and $E(H)$ are the sets of vertices and edges of $H$, respectively. This is a conventional measure of sparseness of an arbitrary graph (not necessary planar). For more details on this invariant see e.g. 8, 13. Dong and Wang first made the link between the maximum average degree and the total neighbour sum distinguishing index. They proved the following result.

Theorem 4 ([10]). Any graph $G$ with $\Delta(G) \geq 5$ and $\operatorname{mad}(G)<3$ satisfies $\chi_{\Sigma}^{\prime \prime}(G) \leq \Delta(G)+2$.

This subject was intensively studied afterwards, and the following improvement has been announced recently (but no proof of such a supposed fact was published thus far).

Theorem 5 ([25]). Any graph $G$ with $\Delta(G) \geq 8$ and $\operatorname{mad}(G)<\frac{9}{2}$ satisfies $\chi_{\Sigma}^{\prime \prime}(G) \leq \Delta(G)+3$.

In this paper, we prove a stronger statement than the above:

Theorem 6. Any graph $G$ with $\Delta(G) \geq 8$ and $\operatorname{mad}(G)<\frac{14}{3}$ satisfies $\chi_{\Sigma}^{\prime \prime}(G) \leq \Delta(G)+3$.

Recall that the girth of a graph is the length of a shortest cycle in it. As every planar graph with girth $g$ satisfies $\operatorname{mad}(G)<\frac{2 g}{g-2}$, the following corollary can be easily derived from Theorem 6 .

Corollary 7. Any triangle-free planar graph $G$ with $\Delta(G) \geq 8$ satisfies $\chi_{\Sigma}^{\prime \prime}(G) \leq \Delta(G)+3$.

\section{Proof of Theorem 6}

\subsection{Preliminaries}

Fix an integer $k \geq 8$. In the following, $n_{i}(G)$ denotes the number of vertices of degree $i$ in a graph $G$ (and similarly for $n_{i^{+}}(G)$ with "at least $i$ " and for $n_{i^{-}}(G)$ with "at most $i$ "). We say a graph $G$ is smaller than a graph $H, G \prec H$ if $|E(G)|+|V(G)|<|E(H)|+|V(H)|$. We say a graph is minimal for a property when no smaller graph verifies it. We shall also call any vertex of degree $d(\geq d, \leq d)$ in a given graph a $d$-vertex $\left(d^{+}\right.$-vertex, $d^{-}$-vertex, resp.) of this graph. The same nomenclature shall be used for neighbours as well. 


\subsection{Structural properties of $H$}

Suppose that $H$ is a minimal graph with maximum degree $\Delta \leq k, \operatorname{mad}(H)<\frac{14}{3}$ and $\chi_{\Sigma}^{\prime \prime}(H)>k+3$ (hence $H$ is connected and $\delta(H) \geq 1$ ). In the remaining part of the paper we argument that in fact $H$ cannot exist, i.e. that there exists a tnsd $(k+3)$-colouring of $H$, and thus prove Theorem 6 ,

In this subsection we exhibit some structural properties of $H$.

The following lemma shall be very useful to this end. Its proof was inspired by the research from 5$]$. The same result but in the case of lists of integers can also be derived from 2].

Lemma 8 ([12]). For any finite sets $L_{1}, \ldots, L_{t}$ of real numbers with $\left|L_{i}\right| \geq t$ for $i=1, \ldots, t$, the set $\left\{x_{1}+\ldots+x_{t}: x_{1} \in L_{1}, \ldots, x_{t} \in L_{t} ; x_{i} \neq x_{j}\right.$ for $\left.i \neq j\right\}$ contains at least $\sum_{i=1}^{t}\left|L_{i}\right|-t^{2}+1$ distinct elements.

Observation 9. Every $3^{-}$-vertex $v$ in $H$ can be recoloured (or coloured if it has no colour assigned) so that it has a different colour than its adjacent vertices and incident edges, and so that $v$ is not in conflict with any of its neighbours.

Proof. This follows directly by the fact we have $k+3 \geq 11$ colours available, while at most 9 of them might be blocked by the requirements from the thesis.

Lemma 10. For every vertex $v \in V(H), n_{4^{+}}(v) \geq n_{2^{-}}(v)+1+n_{3^{-}}(v) \times(k-d(v))$.

Proof. Suppose on the contrary that $v \in V(H)$ with $d(v)=d \geq 1$ is adjacent to $\alpha 2^{-}$-vertices $u_{1}, \ldots, u_{\alpha}, \beta 3$-vertices $w_{1}, \ldots, w_{\beta}$ and to $\gamma 4^{+}$-vertices where $\gamma<\alpha+1+(\alpha+\beta)(k-d)$, hence $\alpha+\beta \geq 1$. Colour $H^{\prime}=H-\left\{v u_{1}, \ldots, v u_{\alpha}, v w_{1}, \ldots, v w_{\beta}\right\}$ by minimality (i.e. fix any tnsd $(k+3)-$ colouring of $H^{\prime}$, which must exist due to the fact that $H$ is our minimal counterexample, while $H^{\prime} \prec H$, $\Delta\left(H^{\prime}\right) \leq k$ and $\left.\operatorname{mad}\left(H^{\prime}\right)<\frac{14}{3}\right)$ and uncolour $u_{i}$ and $w_{j}$ for all $i \in\{1, \ldots, \alpha\}$ and $j \in\{1, \ldots, \beta\}$. Let $L_{i}$ and $L_{j}^{\prime}$, with $i \in\{1, \ldots, \alpha\}$ and $j \in\{1, \ldots, \beta\}$ be the sets of available colours respectively for the edges $v u_{i}$ and $v w_{j}$ (i.e. those colours in $\{1, \ldots, k+3\}$ not used by their adjacent edges in $H$ or $v)$. Note that $\left|L_{1}\right|, \ldots,\left|L_{\alpha}\right| \geq \alpha+\beta+1+k-d$ and $\left|L_{1}^{\prime}\right|, \ldots,\left|L_{\beta}^{\prime}\right| \geq \alpha+\beta+k-d$. By Lemma 8 we may extend this colouring to a (partial) proper colouring of $H$ in different ways, obtaining at least $\alpha(\alpha+\beta)+\alpha+\alpha(k-d)+\beta(\alpha+\beta)+\beta(k-d)-(\alpha+\beta)^{2}+1=\alpha+1+(\alpha+\beta)(k-d)>\gamma$ distinct sums for $v$. Thus we can do it in such a way that $v$ is no in conflict with any of its $4^{+}$-neighbours. By Observation 9 we therefore obtain a contradiction.

Corollary 11. For every vertex $v \in V(H)$ with $d(v) \geq 7, n_{2^{-}}(v) \leq d(v)-5$.

Proof. Suppose to the contrary that $n_{2^{-}}(v) \geq d(v)-4$ for some $v \in V(H)$ with $d(v) \geq 7$. By Lemma 10 we have: $n_{4^{+}}(v) \geq n_{2^{-}}(v)+1+n_{3^{-}}(v) \times(k-d(v)) \geq d(v)-4+1+n_{3^{-}}(v) \times(k-d(v)) \geq d(v)-3$.

Consequently, we have: $d(v) \geq n_{2^{-}}(v)+n_{4^{+}}(v) \geq d(v)-4+d(v)-3=2 d(v)-7$. Hence, $d(v) \leq 7$, i.e. $d(v)=7$. As $k \geq 8$, then by Lemma 10 we thus obtain: $n_{4^{+}}(v) \geq n_{2^{-}}(v)+1+n_{3^{-}}(v) \times(k-d(v)) \geq$ $4+n_{3^{-}}(v) \times(k-d(v)) \geq d(v)$, a contradiction with the fact that $n_{2^{-}}(v) \geq d(v)-4$.

Within the proof of the remaining structural properties of $H$, aggregated in Claim 1 below, we shall apply several times the following algebraic tool due to Alon [2].

Theorem 12 (Combinatorial Nullstellensatz). Let $\mathbb{F}$ be an arbitrary field, and let $P=P\left(x_{1}, \ldots, x_{n}\right)$ be a polynomial in $\mathbb{F}\left[x_{1}, \ldots, x_{n}\right]$. Suppose the coefficient of a monomial $x_{1}^{k_{1}} \ldots x_{n}^{k_{n}}$, where each $k_{i}$ is a nonnegative integer, is non-zero in $P$ and the degree $\operatorname{deg}(P)$ of $P$ equals $\sum_{i=1}^{n} k_{i}$. If moreover $S_{1}, \ldots, S_{n}$ are any subsets of $\mathbb{F}$ with $\left|S_{i}\right|>k_{i}$ for $i=1, \ldots, n$, then there are $s_{1} \in S_{1}, \ldots, s_{n} \in S_{n}$ so that $P\left(s_{1}, \ldots, s_{n}\right) \neq 0$.

Claim 1. The graph $H$ does not contain any of:

(C1) a $2^{-}$-vertex $v$ adjacent to $a\left(\frac{k}{2}+1\right)^{-}$-vertex $u$;

(C2) a $4^{-}$-vertex $v$ adjacent to a $4^{-}$-vertex $u$; 
(C3) a $3^{-}$-vertex $v$ adjacent to a $5^{-}$-vertex $u$;

(C4) a 5-vertex $v$ adjacent to three 4 -vertices $v_{1}, v_{2}, v_{3}$;

(C5) a 6-vertex $v$ adjacent to a $3^{-}$-vertex $u$ and to a $4^{-}$-vertex $w$;

(C6) a 7-vertex $v$ adjacent to a $2^{-}$-vertex $u$, to a $3^{-}$-vertex $w$ and to a $4^{-}$-vertex $y$.

$(C 7)$ a vertex $v$ of degree $d \geq 8$ adjacent to $(d-7) 2^{-}$-vertices $v_{1}, \ldots, v_{d-7}$, to two $3^{-}$-vertices $u_{1}, u_{2}$ and to a $4^{-}$-vertex $w$;

(C8) a vertex $v$ of degree $d=\Delta \geq 3$ adjacent to $(d-2) 3^{-}$-vertices $v_{1}, \ldots, v_{d-2}$ and to one $4^{-}$-vertex $u$.

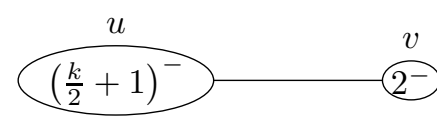

$\left(C_{1}\right)$

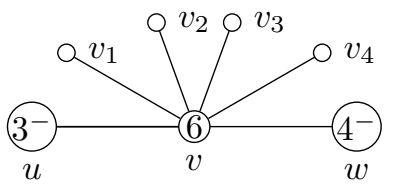

$\left(C_{5}\right)$

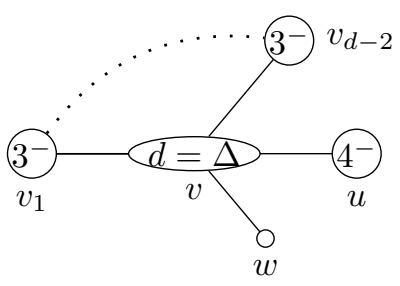

$\left(C_{8}\right)$

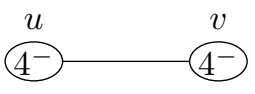

$\left(C_{2}\right)$

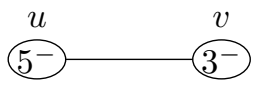

$\left(C_{3}\right)$

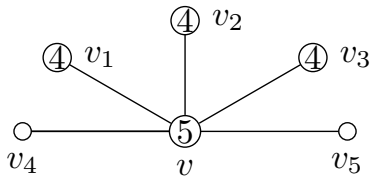

$\left(C_{4}\right)$

$\left(C_{7}\right)$

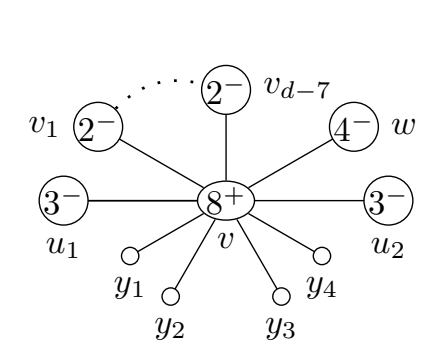

$\left(C_{6}\right)$

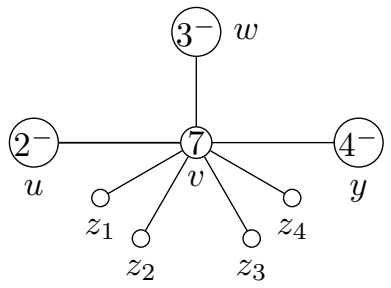

$$
\text { (1) }
$$


is proper we have to avoid at most $\frac{k}{2}+2$ colours, and possibly at most $\frac{k}{2}$ more colours to ensure the sum-distinction (of $u$ from its neighbours other than $v$ ). Hence, we have at least one colour left to extend the colouring, and thus obtain a tnsd $(k+3)$-colouring of $H$ via Observation 9 applied to $v$, a contradiction.

2;3. Suppose there exists an edge $u v$ with $d(u), d(v) \leq 4$ or with $d(u) \leq 5$ and $d(v) \leq 3$ in $H$. Denote the neighbours of $u$ other than $v$ by $u_{1}, \ldots, u_{q}$, and denote the neighbours of $v$ other than $u$ by $v_{1}, \ldots, v_{p}$ (hence $d(u)=q+1$ and $d(v)=p+1)$. By the minimality of $H$ there exists a tnsd $(k+3)$-colouring $c$ of $H^{\prime}=H-\{u v\}$. Let us now undelete the edge $u v$ and remove the colours from $u$ and $v$. In order to extend the current partial colouring of $H$ to its proper total $(k+3)$-colouring we may use at least $11-6=5$ colours for $u$, at least $11-6=5$ colours for $u v$ and at least $11-6=5$ colours for $v$ if $d(u), d(v) \leq 4$, or otherwise: at least 3 colours for $u$, at least 5 colours for $u v$ and at least 7 colours for $v$. Denote the respective lists of available colours by $L_{u}, L_{u v}, L_{v}$, the sum at $u_{i}$ by $s_{i}$ and the sum at $v_{j}$ by $s_{j}^{\prime}$ for $i=1, \ldots, q, j=1, \ldots, p$. Consider a polynomial with real variables:

$$
\begin{aligned}
f\left(x_{0}, x_{1}, x_{2}\right) & =\left(x_{0}-x_{1}\right)\left(x_{0}-x_{2}\right)\left(x_{1}-x_{2}\right)\left(x_{0}+\sum_{i=1}^{q} c\left(u u_{i}\right)-x_{2}-\sum_{i=1}^{p} c\left(v v_{i}\right)\right) \\
& \times \prod_{i=1}^{q}\left(x_{0}+x_{1}+\sum_{j=1}^{q} c\left(u u_{j}\right)-s_{i}\right) \prod_{i=1}^{p}\left(x_{2}+x_{1}+\sum_{j=1}^{p} c\left(v v_{j}\right)-s_{i}^{\prime}\right) .
\end{aligned}
$$

Note that in order to extend the colouring $c$ to a tnsd $(k+3)$-colouring of $H$ it is now sufficient to find a non-zero (i.e. with non-zero value of $f$ ) substitution for $f$ such that $x_{0} \in L_{u}, x_{1} \in L_{u v}$ and $x_{2} \in L_{v}$. It is thus the more sufficient to find a non-zero substitution from these list for the polynomial $g$ defined as $g\left(x_{0}, x_{1}, x_{2}\right):=f\left(x_{0}, x_{1}, x_{2}\right) \cdot\left(x_{0}+x_{1}\right)^{3-q}\left(x_{2}+x_{1}\right)^{3-p}$ if $d(u), d(v) \leq 4$ or by $g\left(x_{0}, x_{1}, x_{2}\right):=f\left(x_{0}, x_{1}, x_{2}\right) \cdot\left(x_{0}+x_{1}\right)^{4-q}\left(x_{2}+x_{1}\right)^{2-p}$ otherwise. In the first of these cases however, the coefficient of the monomial $x_{0}^{4} x_{1}^{3} x_{2}^{3}$ in $g$ is the same as in

$$
h_{1}\left(x_{0}, x_{1}, x_{2}\right)=\left(x_{0}-x_{1}\right)\left(x_{0}-x_{2}\right)^{2}\left(x_{1}-x_{2}\right)\left(x_{0}+x_{1}\right)^{3}\left(x_{2}+x_{1}\right)^{3},
$$

and equals 4 : 2. Analogously, in the second case, the coefficient of the monomial $x_{0}^{2} x_{1}^{3} x_{2}^{5}$ in $g$ is the same as in

$$
h_{2}\left(x_{0}, x_{1}, x_{2}\right)=\left(x_{0}-x_{1}\right)\left(x_{0}-x_{2}\right)^{2}\left(x_{1}-x_{2}\right)\left(x_{0}+x_{1}\right)^{4}\left(x_{2}+x_{1}\right)^{2},
$$

and equals also: 2 . In the both cases we thus obtain a contradiction by the Combinatorial Nullstellensatz.

4. Suppose there exists a 5 -vertex $v$ with $N(v)=\left\{v_{1}, \ldots, v_{5}\right\}$ such that $d\left(v_{1}\right)=d\left(v_{2}\right)=d\left(v_{3}\right)=4$ in $H$. By the minimality of $H$ there exists a tnsd $(k+3)$-colouring $c$ of $H^{\prime}=H-\left\{v v_{1}, v v_{2}, v v_{3}\right\}$. Delete the colours of $v, v_{1}, v_{2}, v_{3}$. We associate variables $x_{0}, x_{1}, x_{2}, x_{3}, x_{4}, x_{5}, x_{6}$ with $v, v v_{1}, v v_{2}, v v_{3}, v_{1}, v_{2}, v_{3}$, respectively. For these we denote the lists of their available colours by $L_{0}, \ldots, L_{6}$ (obtained after excluding from $\{1, \ldots, k+3\}$ the colours already used on their respective adjacent or incident vertices and edges), respectively. Then $\left|L_{0}\right| \geq 7,\left|L_{1}\right|,\left|L_{2}\right|,\left|L_{3}\right| \geq 6,\left|L_{4}\right|,\left|L_{5}\right|,\left|L_{6}\right| \geq 5$. Let

$$
\begin{aligned}
f\left(x_{0}, \ldots, x_{6}\right) & =\left(x_{0}-x_{1}\right)\left(x_{0}-x_{2}\right)\left(x_{0}-x_{3}\right)\left(x_{0}-x_{4}\right)\left(x_{0}-x_{5}\right)\left(x_{0}-x_{6}\right)\left(x_{1}-x_{2}\right)\left(x_{1}-x_{3}\right)\left(x_{2}-x_{3}\right) \\
& \times\left(x_{1}-x_{4}\right)\left(x_{2}-x_{5}\right)\left(x_{3}-x_{6}\right) \prod_{i=1}^{3}\left(x_{0}+x_{1}+x_{2}+x_{3}+s(v)-x_{i}-x_{i+3}-s\left(v_{i}\right)\right) \\
& \times \prod_{i=4}^{5}\left(x_{0}+x_{1}+x_{2}+x_{3}+s(v)-s\left(v_{i}\right)\right) \prod_{i=1}^{3} \prod_{u \in N\left(v_{i}\right) \backslash\{v\}}\left(x_{i}+x_{i+3}+s\left(v_{i}\right)-s(u)\right)
\end{aligned}
$$

\footnotetext{
${ }^{4}$ This and further computations were obtained by means of a computer program; one may verify these using e.g. Wolfram Mathematica.
} 
(where $s(w)$ refers to the contemporary partial sum for every vertex $w$ in $H$ ). Note that the coefficient of the monomial $x_{0}^{6} x_{1}^{5} x_{2} x_{3}^{5} x_{4}^{2} x_{5}^{3} x_{6}^{4}$ in $f$ is the same as in the following polynomial:

$$
\begin{aligned}
g\left(x_{0}, \ldots, x_{6}\right) & =\left(x_{0}-x_{1}\right)\left(x_{0}-x_{2}\right)\left(x_{0}-x_{3}\right)\left(x_{0}-x_{4}\right)\left(x_{0}-x_{5}\right)\left(x_{0}-x_{6}\right)\left(x_{1}-x_{2}\right)\left(x_{1}-x_{3}\right)\left(x_{2}-x_{3}\right) \\
& \times\left(x_{1}-x_{4}\right)\left(x_{2}-x_{5}\right)\left(x_{3}-x_{6}\right)\left(x_{0}+x_{2}+x_{3}-x_{4}\right)\left(x_{0}+x_{1}+x_{3}-x_{5}\right)\left(x_{0}+x_{1}+x_{2}-x_{6}\right) \\
& \times\left(x_{0}+x_{1}+x_{2}+x_{3}\right)^{2}\left(x_{1}+x_{4}\right)^{3}\left(x_{2}+x_{5}\right)^{3}\left(x_{3}+x_{6}\right)^{3},
\end{aligned}
$$

and equals 16. By the Combinatorial Nullstellensatz we thus may extend our colouring to a tnsd $(k+3)$-colouring of $H$, a contradiction.

5. Suppose there exists a 6 -vertex $v$ adjacent to a $3^{-}$-vertex $u$, to a $4^{-}$-vertex $w$ and to vertices $v_{1}, v_{2}, v_{3}, v_{4}$ in $H$. By the minimality of $H$ there exists a tnsd $(k+3)$-colouring $c$ of $H^{\prime}=H-\{v u, v w\}$. Delete the colours of $u, v, w$ and associate variables $x_{0}, x_{1}, x_{2}, x_{3}, x_{4}$ with $v, v u, v w, u, w$, respectively. Denote the lists of available colours for these by $L_{0}, \ldots, L_{4}$, resp., and note that $\left|L_{0}\right| \geq 3,\left|L_{1}\right| \geq 5,\left|L_{2}\right| \geq$ $4,\left|L_{3}\right| \geq 7,\left|L_{4}\right| \geq 5$. Consider a polynomial:

$$
\begin{aligned}
f\left(x_{0}, \ldots, x_{4}\right) & =\left(x_{0}-x_{1}\right)\left(x_{0}-x_{2}\right)\left(x_{0}-x_{3}\right)\left(x_{0}-x_{4}\right)\left(x_{1}-x_{2}\right)\left(x_{1}-x_{3}\right)\left(x_{2}-x_{4}\right) \\
& \times\left(x_{0}+x_{2}+s(v)-x_{3}-s(u)\right)\left(x_{0}+x_{1}+s(v)-x_{4}-s(w)\right) \\
& \times \prod_{i=1}^{4}\left(x_{0}+x_{1}+x_{2}+s(v)-s\left(v_{i}\right)\right) \\
& \times \prod_{y \in N(u) \backslash\{v\}}\left(x_{1}+x_{3}+s(u)-s(y)\right) \prod_{y \in N(w) \backslash\{v\}}\left(x_{2}+x_{4}+s(w)-s(y)\right)
\end{aligned}
$$

and set $g\left(x_{0}, \ldots, x_{4}\right)=f\left(x_{0}, \ldots, x_{4}\right) \cdot\left(x_{1}+x_{3}\right)^{3-d(u)}\left(x_{2}+x_{4}\right)^{4-d(w)}$. Note that the coefficient of the monomial $x_{0}^{2} x_{1}^{4} x_{2}^{3} x_{3}^{5} x_{4}^{4}$ in $g$ is the same as in:

$$
\begin{aligned}
h\left(x_{0}, \ldots, x_{4}\right) & =\left(x_{0}-x_{1}\right)\left(x_{0}-x_{2}\right)\left(x_{0}-x_{3}\right)\left(x_{0}-x_{4}\right)\left(x_{1}-x_{2}\right)\left(x_{1}-x_{3}\right)\left(x_{2}-x_{4}\right) \\
& \times\left(x_{0}+x_{2}-x_{3}\right)\left(x_{0}+x_{1}-x_{4}\right)\left(x_{0}+x_{1}+x_{2}\right)^{4}\left(x_{1}+x_{3}\right)^{2}\left(x_{2}+x_{4}\right)^{3},
\end{aligned}
$$

and equals -10 . By the Combinatorial Nullstellensatz there exists a non-zero substitution for $g$, hence the more for $f$, from the corresponding lists $L_{0}, \ldots, L_{4}$, and thus we may extend our partial colouring to a tnsd $(k+3)$-colouring of $H$, a contradiction.

6. Suppose there exists a 7 -vertex $v$ adjacent to a $2^{-}$-vertex $u$, to a $3^{-}$-vertex $w$ and to a $4^{-}$-vertex $y$ in $H$. Denote the remaining neighbours of $v$ by $z_{1}, z_{2}, z_{3}, z_{4}$. By the minimality of $H$ there exists a tnsd $(k+3)$-colouring $c$ of $H^{\prime}=H-\{v u, v w, v y\}$. Delete the colours of $u, w, y$ and associate variables $x_{1}, x_{2}, x_{3}, x_{4}$ with $v u, v w, v y, y$, respectively. Denote the lists of available colours for these by $L_{1}, L_{2}, L_{3}, L_{4}$, resp., and note that $\left|L_{1}\right| \geq 5,\left|L_{2}\right| \geq 4,\left|L_{3}\right| \geq 3,\left|L_{4}\right| \geq 4$. Consider a polynomial (and note that by Observation 9 we shall be able to colour properly vertices $u$ and $w$ at the end so that these are sum distinguished from their neighbours, thus we omit the corresponding requirements within the polynomial below):

$$
\begin{aligned}
f\left(x_{1}, x_{2}, x_{3}, x_{4}\right) & =\left(x_{1}-x_{2}\right)\left(x_{1}-x_{3}\right)\left(x_{2}-x_{3}\right)\left(x_{3}-x_{4}\right) \prod_{i=1}^{4}\left(x_{1}+x_{2}+x_{3}+s(v)-s\left(z_{i}\right)\right) \\
& \times\left(x_{1}+x_{2}+s(v)-x_{4}-s(y)\right) \prod_{z \in N(y) \backslash\{v\}}\left(x_{3}+x_{4}+s(y)-s(z)\right) .
\end{aligned}
$$

Let $g\left(x_{1}, x_{2}, x_{3}, x_{4}\right)=f\left(x_{1}, x_{2}, x_{3}, x_{4}\right) \cdot\left(x_{3}+x_{4}\right)^{4-d(y)}$. Note that the coefficient of the monomial $x_{1}^{4} x_{2}^{3} x_{3}^{2} x_{4}^{3}$ in $g$ is the same as in:

$$
h\left(x_{1}, x_{2}, x_{3}, x_{4}\right)=\left(x_{1}-x_{2}\right)\left(x_{1}-x_{3}\right)\left(x_{2}-x_{3}\right)\left(x_{3}-x_{4}\right)\left(x_{1}+x_{2}+x_{3}\right)^{4}\left(x_{1}+x_{2}-x_{4}\right)\left(x_{3}+x_{4}\right)^{3},
$$

and equals -6 . Therefore, analogously as above we may extend our colouring, first to $v u, v w, v y, y$ by the Combinatorial Nullstellensatz, and then to $u$ and $w$ by Observation 9, to a tnsd $(k+3)$-colouring of $H$, a contradiction. 
7. Suppose there exists a vertex $v$ of degree $d \geq 8$ adjacent to $(d-7) 2^{-}$-vertices $v_{1}, \ldots, v_{d-7}$, to two $3^{-}$-vertices $u_{1}, u_{2}$ and to a $4^{-}$-vertex $w$ in $H$. The remaining neighbours of $v$ we denote by $y_{1}, y_{2}, y_{3}, y_{4}$. By the minimality of $H$ there exists a tnsd $(k+3)$-colouring $c$ of $H^{\prime}=$ $H-\left\{v v_{1}, v u_{1}, v u_{2}, v w\right\}$. Delete the colours of $v, v_{1}, \ldots, v_{d-7}, u_{1}, u_{2}, w$ and associate variables $x_{0}, x_{1}, x_{2}, x_{3}, x_{4}, x_{5}$ with $v, v v_{1}, v u_{1}, v u_{2}, v w, w$, respectively. Denote the lists of available colours for these by $L_{0}, \ldots, L_{5}$, resp., and note that $\left|L_{0}\right| \geq 3,\left|L_{1}\right| \geq 6,\left|L_{2}\right|,\left|L_{3}\right| \geq 5,\left|L_{4}\right| \geq 4,\left|L_{5}\right| \geq 5$. Consider a polynomial (the vertices $v_{1}, \ldots, v_{d-7}, u_{1}, u_{2}$ shall be coloured at the end via Observation 9):

$$
\begin{aligned}
f\left(x_{0}, \ldots, x_{5}\right) & =\left(x_{0}-x_{1}\right)\left(x_{0}-x_{2}\right)\left(x_{0}-x_{3}\right)\left(x_{0}-x_{4}\right)\left(x_{0}-x_{5}\right)\left(x_{1}-x_{2}\right)\left(x_{1}-x_{3}\right)\left(x_{1}-x_{4}\right) \\
& \times\left(x_{2}-x_{3}\right)\left(x_{2}-x_{4}\right)\left(x_{3}-x_{4}\right)\left(x_{4}-x_{5}\right) \prod_{i=1}^{4}\left(x_{0}+x_{1}+x_{2}+x_{3}+x_{4}+s(v)-s\left(y_{i}\right)\right) \\
& \times\left(x_{0}+x_{1}+x_{2}+x_{3}+s(v)-x_{5}-s(w)\right) \prod_{z \in N(w) \backslash\{w\}}\left(x_{4}+x_{5}+s(w)-s(z)\right) .
\end{aligned}
$$

Let $g\left(x_{0}, \ldots, x_{5}\right)=f\left(x_{0}, \ldots, x_{5}\right) \cdot\left(x_{4}+x_{5}\right)^{4-d(w)}$. Then the coefficient of the monomial $x_{0} x_{1}^{5} x_{2}^{4} x_{3}^{3} x_{4}^{3} x_{5}^{4}$ in $g$ is the same as in:

$$
\begin{aligned}
h\left(x_{0}, \ldots, x_{5}\right) & =\left(x_{0}-x_{1}\right)\left(x_{0}-x_{2}\right)\left(x_{0}-x_{3}\right)\left(x_{0}-x_{4}\right)\left(x_{0}-x_{5}\right)\left(x_{1}-x_{2}\right)\left(x_{1}-x_{3}\right)\left(x_{1}-x_{4}\right) \\
& \times\left(x_{2}-x_{3}\right)\left(x_{2}-x_{4}\right)\left(x_{3}-x_{4}\right)\left(x_{4}-x_{5}\right)\left(x_{0}+x_{1}+x_{2}+x_{3}+x_{4}\right)^{4} \\
& \times\left(x_{0}+x_{1}+x_{2}+x_{3}-x_{5}\right)\left(x_{4}+x_{5}\right)^{3} .
\end{aligned}
$$

and equals 5. By the Combinatorial Nullstellensatz and Observation 9 we may thus extend our partial colouring to a tnsd $(k+3)$-colouring of $H$, a contradiction.

8. Suppose $v$ is a vertex of degree $d=\Delta \geq 3$ adjacent to $(d-2) 3^{-}$-vertices $v_{1}, \ldots, v_{d-2}$ and to one $4^{-}$-vertex $u$ in $H$. Denote the remaining neighbour of $v$ by $w$. By the minimality of $H$ there exists a tnsd $(k+3)$-colouring $c$ of $H^{\prime}=H-\left\{v v_{1}, \ldots, v v_{d-2}, v u\right\}$. Delete the colours of $v, v_{1}, \ldots, v_{d-2}, u$. First extend such a partial colouring of $H$ by choosing a colour (in $\{1, \ldots, k+3\}$ ) for $v$ in the following manner. If $d\left(v_{1}\right)=3$, denote the colours associated to the edges incident with $v_{1}$ and different from $v v_{1}$ by $a$ and $b$, and if $c(v w) \notin\{a, b\}$, choose for $v$ any colour in $\{a, b\} \backslash\{c(w)\}$. In all other cases, choose for $v$ any colour distinct from $c(v w)$ and $c(w)$. Denote the colour of $v$ by $c(v)$. Then choose a colour $c(u)$ for $u$ as small as possible (and note that as our total colouring must be proper, this implies that either $u$ or some of its incident edges other than $u v$ has now colour at most 5 ). Next we choose any colour $c(u v)$ so that the obtained (partial) colouring of $H$ is proper and $u$ is sum distinguished from its neighbours other than $v$ (this is possible, as $k+3>9$ ). Then we subsequently choose greedily colours for $v v_{2}, \ldots, v v_{d-2}$ so that the obtained partial total colouring of $H$ is proper. Finally we choose a colour $c\left(v v_{1}\right)$ for $v v_{1}$ distinct from the colours of its incident edges and the colour of $v$ (by our choice of $c(v)$, this blocks at most $\Delta+1$ choices) so that the sum at $v$ is distinct from the sum at $w$, and if $\Delta \leq k-1$, also distinct from the sum at $u$. We complete our colouring by choosing the colours for $v_{1}, \ldots, v_{d-2}, u$ consistently with Observation 9 . In order to see that the obtained colouring of $H$ is sum distinguishing it is sufficient to note that the sum at $v$ is distinct from the sum at $u$ when $\Delta=k$. Indeed, the sum of colours incident with $v$ except for the colour of $u v$ equals at least $1+\ldots+k=k(k+1) / 2$, while by our choice of the colour for $u$, the sum of its incident colours except the one of $u v$ is at most $5+(k+3)+(k+2)+(k+1)=3 k+11<k(k+1) / 2$ (for $k \geq 8)$. Thus we obtain a contradiction with the minimality of $H$.

\subsection{Discharging procedure}

In this subsection we use the discharging technique exploiting the vertices of the graph $H$. For this aim we first define the weight function $\omega: V(H) \rightarrow \mathbb{R}$ by setting $\omega(x)=d(x)-\frac{14}{3}$ for every $x \in V(H)$. Next we shall apply so called Ghost vertices method, introduced earlier by Bonamy, Bousquet and Hocquard [4], and based on the following observation (where given any subsets $U, U^{\prime} \subseteq V(H)$ and a vertex $v, d_{U}(v)$ denotes the number of neighbours of $v$ from $U$, while $E\left(U, U^{\prime}\right)$ is the set of edges joining $U$ and $U^{\prime}$ in the graph $H$ ). 
Observation 13. Let $V_{1} \cup V_{2}$ be a partition of $V(H)$ where, say $V_{1}$ is the set of vertices of degree at least 3 and $V_{2}$ - the set of vertices of degree at most 2 in $H$;

- every vertex $u$ in $H$ has an initial weight $w(u)=d(u)-\frac{14}{3}$.

- If we can discharge the weights in $H$ so that:

1. every vertex in $V_{1}$ has a non-negative weight;

2. and every vertex $u$ in $V_{2}$ has a final weight of at least $d(u)-\frac{14}{3}+d_{V_{1}}(u)$, then

for the new weight assignment $\omega^{\prime}$, we have $\sum_{v \in V_{2}}\left(d(v)-\frac{14}{3}+d_{V_{1}}(v)\right) \leq \sum_{v \in V_{2}} \omega^{\prime}(v)$, as well as $\sum_{v \in V} \omega(v)=\sum_{v \in V} \omega^{\prime}(v)$ and $\sum_{v \in V_{1}} \omega^{\prime}(v) \geq 0$. Therefore,

$$
\begin{aligned}
\sum_{v \in V_{1}}\left(d_{V_{1}}(v)-\frac{14}{3}\right) & \geq \sum_{v \in V_{1}}\left(d_{V_{1}}(v)-\frac{14}{3}\right)+\sum_{v \in V_{2}}\left(d(v)-\frac{14}{3}+d_{V_{1}}(v)\right)-\sum_{v \in V_{2}} \omega^{\prime}(v) \\
& \geq \sum_{v \in V_{1}}\left(d_{V_{1}}(v)-\frac{14}{3}\right)+\left|E\left(V_{1}, V_{2}\right)\right|+\sum_{v \in V_{2}}\left(d(v)-\frac{14}{3}\right)-\sum_{v \in V_{2}} \omega^{\prime}(v) \\
& \geq \sum_{v \in V_{1}}\left(d(v)-\frac{14}{3}\right)+\sum_{v \in V_{2}}\left(d(v)-\frac{14}{3}\right)-\sum_{v \in V_{2}} \omega^{\prime}(v) \\
& \geq \sum_{v \in V} \omega(v)-\sum_{v \in V_{2}} \omega^{\prime}(v) \\
& \geq \sum_{v \in V_{1}} \omega^{\prime}(v) \\
& \geq 0 .
\end{aligned}
$$

Thus we can conclude that $\operatorname{mad}(H) \geq \operatorname{mad}\left(H\left[V_{1}\right]\right) \geq \frac{14}{3}$.

In other words, the vertices in $V_{2}$ can be seen but, in a way, do not contribute to the sum analysis.

In order to finish the proof of Theorem [6 it suffices to obtain a contradiction, e.g. with the fact that $\operatorname{mad}(H)<\frac{14}{3}$, implying that in fact no counterexample to its thesis may exist. By Observation [13] it is thus enough to redistribute the weight (defined by $\omega$ above) in $H$ so that every vertex of degree at least 3 has a non-negative resulting weight, every vertex of degree 2 has weight at least $2-\frac{14}{3}+2$ and every vertex of degree 1 has weight at least $1-\frac{14}{3}+1$.

The discharging rules we shall use for this aim are defined as follows:

(R1) A vertex of degree $d \geq 6$ gives 1 to every adjacent 1-vertex and to every adjacent 2-vertex.

(R2) A vertex of degree $d \geq 6$ gives $\frac{5}{9}$ to every adjacent 3 -vertex.

(R3) A vertex of degree $d \geq 5$ gives $\frac{1}{6}$ to every adjacent 4 -vertex.

Let $v$ be a vertex in $H$. We consider different cases depending on the degree of $v$.

- Assume $d(v)=1$. By $(C 1), v$ is adjacent to a vertex of degree at least 6 . Thus, by $(R 1), v$ receives 1. So every vertex of degree 1 in $H$ has an initial weight of $-\frac{11}{3}$, gives nothing according to our rules and receives 1 , hence has the final weight of $-\frac{8}{3}$.

- Assume $d(v)=2$. By $(C 1), v$ is adjacent to two vertices of degree at least 6 , and thus receives $2 \times 1$ by $(R 1)$ and gives away nothing according to the rules above. So every vertex of degree 2 in $H$ has an initial weight of $-\frac{8}{3}$ and has the final weight of $-\frac{2}{3}$. 
- Assume $d(v)=3$. By $(C 3), v$ is adjacent to three vertices of degree at least 6 , and thus receives $3 \times \frac{5}{9}$ by $(R 2)$ and gives away nothing according to the rules above. So every vertex of degree 3 in $H$ has the final weight 0 .

- Assume $d(v)=4$. By $(C 2), v$ is adjacent to four vertices of degree at least 5 , and thus receives $4 \times \frac{1}{6}$ by $(R 3)$ and gives away nothing according to the rules above. So every vertex of degree 4 in $H$ has the final weight 0 .

- Assume $d(v)=5$. By $(C 3), v$ is not adjacent to a vertex with degree at most 3 , and by $(C 4)$, $v$ is adjacent to at most two vertices of degree 4 , and thus gives at most $2 \times \frac{1}{6}$ by (R3). Hence, $\omega^{\prime}(v) \geq 5-\frac{14}{3}-2 \times \frac{1}{6}=0$.

- Assume $d(v)=6$. Then consider the following subcases:

- if $v$ is adjacent to a $3^{-}$-vertex, then by $(C 5), v$ is adjacent to five vertices of degree at least 5 . Hence, by $(R 1)$ and $(R 2), \omega^{\prime}(v) \geq 6-\frac{14}{3}-1 \times \max \left\{1 ; \frac{5}{9}\right\}=\frac{1}{3} \geq 0$.

- if $v$ is not adjacent to a $3^{-}$-vertex, then $v$ is adjacent to at most six 4 -vertices. Hence, by $(R 3)$, $\omega^{\prime}(v) \geq 6-\frac{14}{3}-6 \times \frac{1}{6}=\frac{1}{3} \geq 0$. In both cases $v$ has a non-negative final weight.

- Assume $d(v) \geq 7$. Recall that by Corollary 11, $n_{2^{-}}(v) \leq d(v)-5$. Then consider the following subcases:

- If $n_{2^{-}}(v)=d(v)-5$ then by $(C 6)$ and $(C 7), v$ is not adjacent to another $4^{-}$-vertex. Hence, by $(R 1), \omega^{\prime}(v) \geq d(v)-\frac{14}{3}-(d(v)-5) \times 1 \geq 0$.

- If $n_{2^{-}}(v)=d(v)-6$ then either $v$ is adjacent to no 3 -vertex or, by $(C 6)$ and $(C 7)$, to exactly one 3 -vertex and to no 4 -vertices. Hence, by $(R 1),(R 2)$ and $(R 3), \omega^{\prime}(v) \geq d(v)-\frac{14}{3}-(d(v)-6) \times$ $1-\max \left\{6 \times \frac{1}{6} ; 1 \times \frac{5}{9}\right\} \geq 0$.

- If $n_{2^{-}}(v)=d(v)-7$ then $v$ is adjacent to at most three 3 -vertices; for $d(v) \geq 8$ it follows by $(C 7)$, while for $d(v)=7$ by Lemma 10, which implies then that $n_{4^{+}}(v) \geq 1+n_{3^{-}}(v)(k-d(v)) \geq$ $1+n_{3^{-}}(v)$. Hence, by $(R 1),(R 2)$ and $(R 3), \omega^{\prime}(v) \geq d(v)-\frac{14}{3}-(d(v)-7) \times 1-3 \times \frac{5}{9}-4 \times \frac{1}{6} \geq 0$.

- If $n_{2^{-}}(v) \leq d(v)-8$ then by $(C 8)$ :

$*$ if $v$ is not adjacent to any 4-vertex then $v$ is adjacent to $(d(v)-8-\alpha) 2^{-}$-vertices and to at most $(\alpha+6) 3$-vertices for some $\alpha \geq 0$. Hence, by $(R 1),(R 2)$ and $(R 3), \omega^{\prime}(v) \geq$ $d(v)-\frac{14}{3}-(d(v)-8-\alpha) \times 1-(\alpha+6) \times \frac{5}{9}=\frac{4}{9} \alpha \geq 0$.

$*$ if $v$ is adjacent to at least one 4 -vertex then $v$ is adjacent to $(d(v)-8-\alpha) 2^{-}$-vertices, to $(\alpha+6-\beta) 3$-vertices and to at most $(\beta+2) 4$-vertices for some $\alpha \geq 0$ and some $\beta \geq 1$. Hence, by $(R 1),(R 2)$ and $(R 3), \omega^{\prime}(v) \geq d(v)-\frac{14}{3}-(d(v)-8-\alpha) \times 1-(\alpha+6-\beta) \times \frac{5}{9}-(\beta+2) \times \frac{1}{6}=$ $\frac{4}{9} \alpha+\frac{7}{18} \beta-\frac{1}{3} \geq 0$.

In all cases $v$ has a non-negative final weight.

This, by Observation 13 , completes the proof of Theorem 6 .

\section{References}

[1] M. Aigner, E Triesch, Irregular assignments of trees and forests., SIAM J. Discrete Math. 3 (1990) $439-449$.

[2] N. Alon, Combinatorial Nullstellensatz, Combin. Probab. Comput. 8 (1999) 7-29.

[3] M. Behzad, Graphs and Their Chromatic Numbers, Ph.D. Thesis, Michigan State University (1965).

[4] M. Bonamy, N. Bousquet, H. Hocquard, Adjacent vertex-distinguishing edge coloring of graphs, Proc. of The Seventh European Conference on Combinatorics, Graph Theory and Applications (2013) 313-318.

[5] M. Bonamy, J. Przybyło, On the Neighbor Sum Distinguishing Index of Planar Graphs, J. Graph Theory 85(3) (2017) $669-690$.

[6] G. Chartrand, P. Erdős, O.R. Oellermann, How to Define an Irregular Graph, College Math. J. 19(1) (1988) 36-42.

[7] G. Chartrand, M.S. Jacobson, J. Lehel, O.R. Oellermann, S. Ruiz, F. Saba, Irregular networks, Congr. Numer. 64 (1988) 197-210. 
[8] N. Cohen, Several Graph problems and their Linear Program formulations, (2010).

[9] B. Cuckler, F. Lazebnik, Irregularity Strength of Dense Graphs, J. Graph Theory 58(4) (2008) 299-313.

[10] A. Dong, G. Wang, Neighbor sum distinguishing total colorings of graphs with bounded maximum average degree, Acta Matematica Sinica 30(4) (2014), 703-709.

[11] A. Frieze, R.J. Gould, M. Karoński, F. Pfender, On Graph Irregularity Strength, J. Graph Theory 41(2) (2002) $120-137$.

[12] H. Hocquard, J. Przybyło, On the neighbour sum distinguishing index of graphs with bounded maximum average degree, Graphs and Combinatorics 33(6) (2017) 1459-1471.

[13] T.R. Jensen, B. Toft, Choosability versus chromaticity, Geombinatorics 5 (1995) 45-64.

[14] M. Kalkowski, M. Karoński, F. Pfender, A new upper bound for the irregularity strength of graphs, SIAM J. Discrete Math. 25(3) (2011) 1319-1321.

[15] J. Lehel, Facts and quests on degree irregular assignments, Graph Theory, Combinatorics and Applications, Willey, New York, 1991, 765-782.

[16] H. Li, L. Ding, B. Liu, G. Wang, Neighbor sum distinguishing total colorings of planar graphs, J. Comb. Optim. 30(3) (2015) 675-688.

[17] H. Li, B. Liu, G. Wang, Neighbor sum distinguishing total colorings of $K_{4}$-minor free graphs, Frontiers of Mathematics in China 8(6) (2013) 1351-1366.

[18] M. Molloy, B. Reed, A bound on the Total Chromatic Number, Combinatorica 18 (1998) 241-280.

[19] S. Loeb, J. Przybyło, Y. Tang, Asymptotically optimal neighbor sum distinguishing total colorings of graphs, Discrete Math. 340(2) (2017) 58-62.

[20] P. Majerski, J. Przybyło, On the irregularity strength of dense graphs, SIAM J. Discrete Math. 28(1) (2014) 197-205.

[21] T. Nierhoff, A tight bound on the irregularity strength of graphs, SIAM J. Discrete Math. 13 (2000) 313-323.

[22] M. Pilśniak, M. Woźniak, On the Total-Neighbor-Distinguishing Index by Sums, Graphs Combin. 31(3) (2015) 771-782.

[23] J. Przybyło, A note on asymptotically optimal neighbour sum distinguishing colourings, submitted.

[24] J. Przybyło, Neighbour distinguishing total colourings via the Combinatorial Nullstellensatz, Discrete Appl. Math. 202 (2016) 163-173.

[25] B. Qiu, J. Wang, Y. Liu, Z. Xu, Neighbor sum distinguishing total colorings of graphs with bounded maximum degree and maximum average degree, CSE 2017 IEEE Conference Publications 1 (2017) 898-901, http://ieeexplore.ieee.org/document/8005933

[26] V. Vizing, Some Unsolved Problems in Graph Theory, Russian Math Surveys 23 (1968) 125-141.

[27] D. Yang, L. Sun, X. Yu, J. Wu, S. Zhou, Neighbor sum distinguishing total chromatic number of planar graphs with maximum degree 10, Applied Mathematics and Computation 314 (2017) 456-468. 